\title{
ROBUST SIGNATURE-BASED GEOMETRIC INVARIANT COPYRIGHT PROTECTION
}

\author{
Mohammad Awrangjeb and Manzur Murshed
}

\author{
Gippsland School of Information Technology, Monash University, Churchill Vic 3842, Australia \\ \{Mohammad.Awrangjeb, Manzur.Murshed\}@infotech.monash.edu.au
}

\begin{abstract}
The most significant bit (MSB)-plane of an image is least likely to change by the most signal processing operations. Watermarking techniques are, however, unable to exploit the MSB-plane, as embedding any information there introduces the highest distortion. This paper presents a novel rotation, scale, and translation (RST)resistant multi-bit logo-based copyright protection scheme using the most significant gray-scale bits at the region-of-interest, automatically selected by the invariant centroid (IC) of the image. How the RST-attack can be reversed using the IC and geometric moments has been proposed. During verification, the test image is restored to its approximate original through reversing any possible RST attack before calculating signature. To avoid any bias, a new MSB-based attack has also been proposed. Experimental results have clearly demonstrated the superiority of the proposed scheme.
\end{abstract}

Index Terms-Copyright protection, signal processing, distortion

\section{INTRODUCTION}

With the ease of multimedia communications using the Internet and the increase in freely available image editing tools, enforcement of image copyright protection has become more imperative than ever. Although digital watermarking has gained considerable attention [1-3], the robustness of this technique is questionable to survive geometric distortions, counterfeit, collusion and copy attacks. No watermarking scheme can resist all kinds of attacks; while schemes in [1-2] are suitable for resisting rotation, scale, and translation (RST) attacks, the scheme in [3] is suitable for resisting highly lossy compression. In addition, distorting the original image is unacceptable for some applications e.g., medical imagery and satellite data [4]. Cryptographic tools, on the contrary, can protect the content with very high security [5]; but these are useless to protect information in the Internet.

The recent signature-based distortion-free copyright protection technique [5-9] combines advantages of both digital watermarking and cryptography. This technique, in general, calculates a digital signature of an image using a logo which is then signed by a certification authority (CA) and protected using the public key cryptography. It can offer cryptographic security; while surviving many of the abovementioned difficult attacks to demonstrate reasonable robustness. In addition, joint ownership can be easily implemented with multiple logos.

Among the existing signature-based schemes, Lee and Chen [9] calculated the signature of an image with a gray-scale logo using vector quantization (VQ) on the coarse scale of the image obtained by a $t$-scale wavelet transform. Chen et al. [5] later extended this idea for binary logos by replacing VQ with a polarity table. However, the size of the coarse image reduces exponentially as $t$ increases. Compounded with the approximation due to VQ, this can potentially lead to very poor quality of the retrieved logo, especially when the original image size is small as demanded by the WWW. In addition, uniqueness of the signature, where it should verify the corresponding image only, may not be guaranteed with binary logos. Chang et al. [7], at the same time, calculated signature with a gray-scale logo using torus automorphism functions. To survive the cropping attacks, the idea of using a rectangular region-of-interest (ROI) in the image was introduced in [6]. Nevertheless, it still cannot survive in rotation and cropping attacks. Moreover, high computational complexity of all these existing schemes limits their real time applications.

In this paper, we propose a computationally inexpensive signature-based gray-scale image copyright protection scheme intuitively using the most significant bit (MSB)-plane, which is least likely to change by any image processing operation. Besides being image size invariant, this ROI-based scheme can be used with any $n$-bit logo. The automatic selection of the ROI is done using the invariant centroid (IC) [10] of the image. The IC can be always detected under any RST attack, even if the image is cropped [10]. To resist RST attacks, we propose how we can reverse the operation using the IC [10] and geometric moments [11]. In verification phase, the tested image is restored to its approximate original through reversing any possible RST attack before calculating the signature. To avoid any bias, we further propose a new MSB-based attack, which would defeat our scheme most. Experimental results show very high logo retrieval rate and visual quality of the retrieved logos by the proposed scheme against those by the Torus-based [7] and VQ-based [9] schemes.

\section{MOTIVATION}

In order to establish the motivation of using the MSB-plane for digital signature, we conducted experiments on a large database of 1032 images [12], including the benchmark ones [13]. Fig. 1(a) shows that on average more than $91 \%$ of the gray-scale MSBs remained the same even when JPEG quality was set at the minimum; while more than $85 \%$ of the MSBs remained unchanged if the image is rotated by $\pm 5^{\circ}$. If we could reverse the rotation, the MSB similarity rate would be increased. We further observed that under median filter $(3 \times 3$ window), histogram equalization, salt $\&$ pepper noise and Gaussian noise attacks on average more than $94 \%, 80 \%, 98 \%$, and $94 \%$ of MSBs respectively were unchanged.

In order to avoid any bias, we now propose a new attack, namely the MSB attack, where for a given target image-quality (in PSNR), the maximum number of gray-scale MSBs are changed. Fig. 1(b) shows that on average more than $80 \%$ of the MSBs, with no more than $10 \%$ standard deviation, remained unchanged at 30 $\mathrm{dB}$ target PSNR, below which the visual quality of the image is unacceptable to the human eyes [9]. 


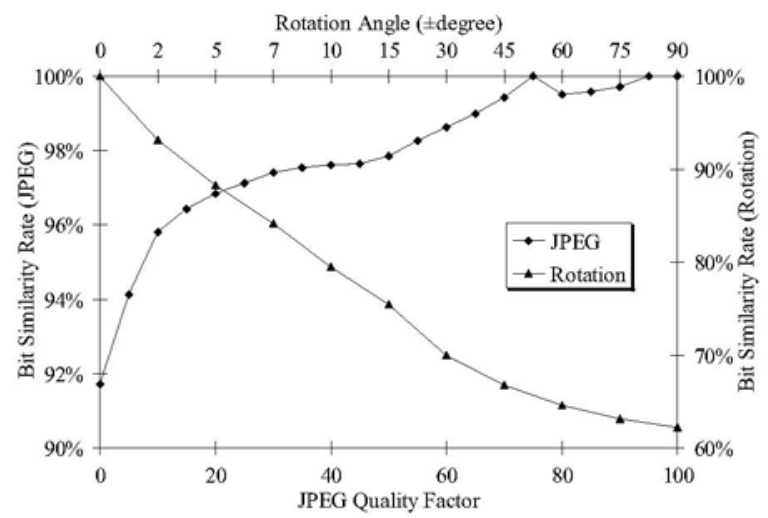

(a)

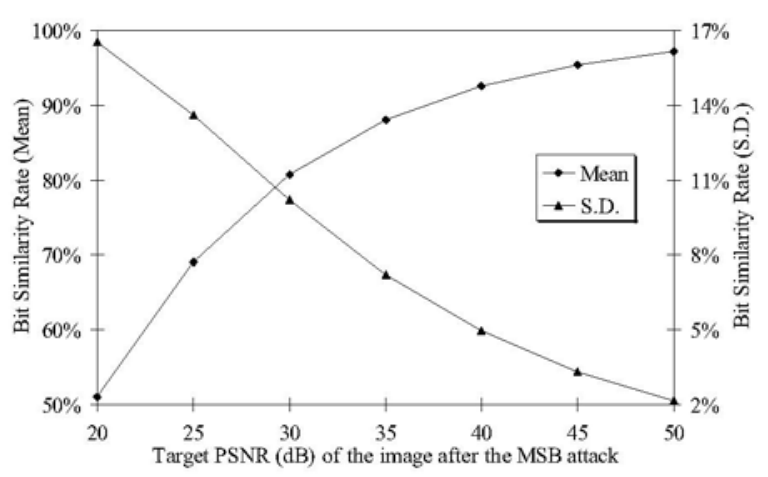

(b)

Fig. 1: (a) Similarity rates of gray-scale MSB at different JPEG and rotation attacks; (b) mean and standard deviation of gray-scale MSB similarity under the newly proposed MSB attack. Note that the rotational angle axis in (a) is non-linear

\section{THE PROPOSED SCHEME}

The proposed scheme first calculates digital signature of an image for a logo at an automatically selected ROI. This signature is then signed by the CA after adding the current time-stamp TS. During verification, the signature may be calculated after reversing any possible RST attack.

\section{1. Automatic ROI Selection}

Consider an $n$-bit gray-scale image $I=\left\{i_{b}(x, y)\right\}$ of size $w_{I} \times h_{I}$ pixels where $1 \leq x \leq w_{I}, \quad 1 \leq y \leq h_{I}$, and $1 \leq b \leq n$. Similarly consider an $n$-bit gray-scale $\log L=\left\{l_{b}(x, y)\right\}$ of size $w_{L} \times h_{L}$. We first pass $I$ through Gaussian low pass filter which reduces the effect of waveform attacks like JPEG, noise addition etc. Then the IC $C_{I}=\left(c_{x}, c_{y}\right)$ of $I$ is obtained using the scheme proposed in [10]. A rectangular area $R_{I}=(t l, b r)$, centered at $C_{I}$, is selected as the ROI, where $t l=\left(x_{1}, y_{1}\right)$ and $b r=\left(x_{2}, y_{2}\right)$ denote the top-left and bottom-right coordinates of the rectangle with respect to $I$.

\section{2. Signature Calculation}

Let $R_{I, M S B}=\left\{r_{j} \in\left\{i_{n}\right\}\right\}$ be the ordered collective set of MSBs in $I$ at $R_{I}$ where $1 \leq j \leq\left(x_{2}-x_{1}+1\right)\left(y_{2}-y_{1}+1\right)$. Without any loss of generality, it is assumed that

$$
\left(x_{2}-x_{1}+1\right)\left(y_{2}-y_{1}+1\right) \geq n w_{L} h_{L} .
$$

MSB-based signature $S_{I L}$ of $I$ for $L$ at $R_{I}$ is thus calculated as

$$
S_{I L}\left(j=(b-1) w_{L} h_{L}+(y-1) h_{L}+x\right)=r_{j} \oplus l_{b}(x, y) .
$$

If the generality assumption in (1) cannot be met, $r_{j}$ 's could be reused iteratively once exhausted. Moreover, any colour image can

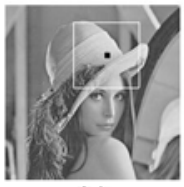

(a)

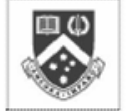

(b)

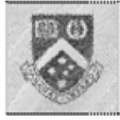

(c)

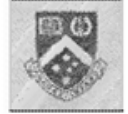

(d)
Fig. 2: (a) Original Lena image $(512 \times 512,8$-bit gray) showing its invariant centroid and ROI, (b) Original Monash logo $(64 \times 64,8$-bit gray), (c) mapped logo ( $15.86 \mathrm{~dB}, 56 \%)$ by Torus-based scheme [7], and (d) VQ coded logo $(23.40,64 \%)$ by VQ-based scheme [9] be signed using its gray-scale equivalent with even a coloured logo after stripping it into three gray-scale channels.

Once the signature is calculated, the owner sends the following message, in the form of an encrypted signature-triplet, to the CA using public key cryptography:

$$
M=E_{T, P U B}\left(E_{O, P R V}\left(\left[I, L, S_{I L}\right]\right)\right)
$$

where $E_{T, P U B}$ and $E_{O P R V}$ are the public and private key encryptions of the CA and the owner respectively. The CA verifies $S_{I L}$ for $I$ and $L$, appends $T S$ with $S_{L L}$, and sends back the following message to the owner:

$$
M^{\prime}=E_{O, P U B}\left(E_{T, P R V}\left(S_{I L} \| T S\right)\right)
$$

where $E_{T, P R V}$ and $E_{O, P U B}$ are the private and public key encryptions of the CA and the owner respectively.

The owner either stores the signature in the database or adds as a part of the image header as in $[5,9]$ allowing public verification.

\section{3. Reversing RST attacks using Geometric Invariance}

Define the geometric moments $m_{p, q}$ of a grayscale image $I(x, y)$ as

$$
m_{p, q}=\sum_{x} \sum_{y} x^{p} y^{q} I(x, y)
$$

and the central moments $\mu_{p, q}$ as

$$
\mu_{p, q}=\sum_{x} \sum_{y}(x-\bar{x})^{p}(y-\bar{y})^{q} I(x, y)
$$

where $(\bar{x}, \bar{y})$ is the centroid of the image defined as

$$
\bar{x}=\frac{m_{1,0}}{m_{0,0}}, \bar{y}=\frac{m_{0,1}}{m_{0,0}} .
$$

The $x$ and $y$-directions in the above equations change when the image is rotated and scaled. In order to estimate these directions, we use the principal axis concept applied to the binary image [11]. The correction angle $\theta_{c}$ can be expressed as

$$
\theta_{c}=\frac{1}{2} \tan ^{-1} \frac{2 \mu_{1,1}}{\mu_{2,0}-\mu_{0,2}} .
$$

The rotated and scaled image should be rotated by $-\theta_{c}$ before measuring the transform parameters. Again define the geometric moments computed from the center $\left(x_{0}, y_{0}\right)$ of the image as 


$$
\tilde{\mu}_{p, q}=\sum_{x} \sum_{y}\left(x-x_{0}\right)^{p}\left(y-y_{0}\right)^{q} I(x, y) .
$$

Flipping can be a part of an RST attack. We achieve flipping normalization using signs of $\widetilde{\mu}_{1,0}$ and $\widetilde{\mu}_{0,1}$ as they change their signs with flipping around $y$ and $x$-axis respectively [11]. The translation invariance is achieved using the IC $C_{I}$ as it is invariant to the center $\left(x_{0}, y_{0}\right)$. In rotation only attacks, the rotated image may or may not be cropped to the original size. If $I$ is rotated by an angle $\theta$, the IC is also rotated by the angle $\theta$, irrespective of the cropping after rotation. Let the IC of the rotated image be $C_{r r}=\left(c_{x r}, c_{y r}\right)$. Then $\theta=\alpha_{r}-\alpha$, where $\alpha_{r}=\tan ^{-1}\left(c_{y r} / c_{x r}\right)$ and $\alpha=\tan ^{-1}\left(c_{y} / c_{x}\right)$. In scale only attacks, If $I$ is scaled by a factor $s$ in both $x$ and $y$ directions, the linear distance of the IC from the center of the image changes by the factor $s$; however, its angular distance $\alpha$ does not change. Let the IC of the scaled image be $C_{I s}=\left(c_{x s}, c_{y s}\right)$. Then $s=R_{s} / R$, where $R_{s}=\sqrt{c_{x s}^{2}+c_{y s}^{2}}$ and $R=\sqrt{c_{x}^{2}+c_{y}^{2}}$. In rotation-

Table I: Comparative logo retrieval results for the proposed MSB-

based scheme and existing Torus-based [7] and VQ-based [9] schemes against various attacks using Lena image and Monash logo

\begin{tabular}{|c|c|c|c|c|c|c|c|}
\hline \multirow{3}{*}{ Attacks } & \multirow{3}{*}{$\begin{array}{c}\text { Image } \\
\text { PSNR } \\
(\mathrm{dB})\end{array}$} & \multirow{2}{*}{\multicolumn{2}{|c|}{$\begin{array}{c}\text { Proposed } \\
\text { scheme }\end{array}$}} & \multicolumn{4}{|c|}{ Existing schemes } \\
\hline & & & & \multicolumn{2}{|c|}{ Torus logo } & \multicolumn{2}{|c|}{ VQ $\log 0$} \\
\hline & & $\begin{array}{c}\text { PSNR } \\
(\mathrm{dB}) \\
\end{array}$ & $\begin{array}{c}\text { LRR } \\
(\%)\end{array}$ & $\begin{array}{c}\text { PSNR } \\
\text { (dB) }\end{array}$ & \begin{tabular}{|c|} 
LRR \\
$(\%)$
\end{tabular} & $\begin{array}{c}\text { PSNR } \\
\text { (dB) }\end{array}$ & \begin{tabular}{|c|} 
LRR \\
$(\%)$
\end{tabular} \\
\hline Affin & .66 & 23.49 & 97 & 14.73 & 54 & 14.79 & 58 \\
\hline Avg. filter ${ }^{1}$ & 5.85 & 31.14 & שנק & 14.97 & 53 & 3.08 & 60 \\
\hline$B P M(25 d B)$ & 2.94 & 19.67 & 95 & 13.85 & 56 & 3.67 & 73 \\
\hline Cropning $175^{\circ}$ & - & 8.24 & & - & - & - & - \\
\hline s. (G) filter ${ }^{1}$ & 36.89 & 31.23 & 99 & 14.97 & 53 & .12 & 60 \\
\hline G filter ${ }^{1} \& \mathrm{G}$ noise & 26.56 & 4.40 & 99 & 14.69 & 55 & & 61 \\
\hline & 23.03 & 22.20 & 97 & 4.52 & 57 & & 66 \\
\hline & 19.36 & 19.93 & 94 & 16.94 & 62 & & 55 \\
\hline ality 0) & 24.88 & 17.40 & 91 & 16.85 & 58 & & 70 \\
\hline JPEG (q & 28.23 & 20.26 & 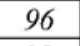 & 17.14 & 50 & & \\
\hline lity 10$)$ & 31.93 & 25.00 & 98 & 17.27 & 56 & & 66 \\
\hline PEG (quality 15 ) & 33.91 & 25.14 & 97 & 7.41 & 55 & & \\
\hline & 20.16 & 18.49 & 92 & & 55 & & 70 \\
\hline & 7.20 & 28.55 & 99 & 15.27 & 53 & & 61 \\
\hline 急 & 2.27 & 18.14 & 91 & & 53 & & 63 \\
\hline Prin & 1.63 & 20.45 & 94 & 3.20 & 61 & 2 & 71 \\
\hline & & .54 & & & 53 & & 69 \\
\hline & .00 & 14.80 & & & 53 & & \\
\hline B) & 5.00 & 19.31 & 90 & 15.16 & 53 & 5 & 62 \\
\hline & 5.00 & 23.37 & & & & & \\
\hline $\mathrm{Rot}$ & & & & & & & 0 \\
\hline & 19.48 & 19.92 & 9. & & 53 & & 64 \\
\hline & .33 & 21.43 & & .37 & 53 & & \\
\hline$(1 \mathrm{HI} 10)$ & & & & & 53 & & 61 \\
\hline & 18.55 & 23.30 & 98 & 15.11 & 53 & 23.04 & 65 \\
\hline $\mathrm{gg}^{4}(\times 0.5)$ & 33.98 & 31.01 & 99 & 12.99 & 53 & 23.41 & 62 \\
\hline Scaling $^{4}(\times 2)$ & 39.34 & 27.93 & 98 & 15.02 & 53 & 23.49 & 66 \\
\hline Self similarities & 26.04 & 18.25 & 94 & 15.16 & 57 & 23.47 & 66 \\
\hline 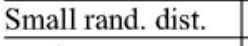 & 16.27 & 19.41 & 90 & 16.71 & 53 & 16.31 & 69 \\
\hline unZign & 29.08 & 24.71 & 98 & 11.55 & 54 & 23.38 & 65 \\
\hline
\end{tabular}

${ }^{1} 3 \times 3$ window; ${ }^{2}$ Latest small rand. dist.; ${ }^{3}$ Row-col-removal; ${ }^{4}$ Resized to the original size $\left(w_{I} \times h_{I}\right.$ pixels $)$. scale attacks, $I$ is first rotated by an angle $\theta_{r s}$ and then scaled down to the original size. Let the rotation-scale attacked image be $I_{r s}$. We follow the steps below in order to reverse the rotation-scale attack: Step 1: Let $I_{g}$ be the Gaussian low pass filtered version of $I_{r s}$. Step 2: Calculate the correction angle $\theta_{c}$ for $I_{g}$. Step 3: Rotate $I_{g}$ by an angle $-\theta_{c}$. Let this corrected image be $I_{g c}$. Step 4: Resize $I_{g c}$ to the original size. Let this resized image be $I_{g c s}$. Step 5: Find the IC $C_{g c s}=\left(c_{x g c s}, c_{y g c s}\right)$ of $I_{g c s}$.

Step 6: Find the IC $C_{g c}=\left(c_{x g c}, c_{y g c}\right)$ of $I_{g c}$ using $C_{g c s}$ and scaling factor between $I_{g c}$ and $I_{g c s}$.

Step 7: Find the IC $C_{g}=\left(c_{x g}, c_{y g}\right)$ of $I_{g}$ by rotating the centroid point $C_{g c}=\left(c_{x g c}, c_{y g c}\right)$ by the angle $\theta_{c}$.

Step 8: Let the distance of $C_{g}$ from the center of $I_{g}$ be $R_{g}$ and that of $C$ from the center of $I$ be $R$.

Step 9: Find the scaling factor $s_{r s}=R_{g} / R$.

Step 10: Resize $I_{g}$ using the scaling factor $1 / s_{r s}$. Let this resized image be $I_{g s}$.

Step 11: Crop $I_{g s}$ to resize it to the original size. Let this resized image be $I_{\text {gscp }}$ which is the cropped rotated image of the original image.

Step 12: Find the IC $C_{g s c p}=\left(c_{x g s c p}, c_{y g s c p}\right)$ of $I_{g s c p}$.

Step 13: Calculate the rotation angle $\theta_{r s}$, between $C$ and $C_{g s c p}$.

Step 14: Resize and rotate the input image $I_{r s}$ by the factor $1 / s_{r s}$ and the angle $-\theta_{r s}$. Let this scaled and rotated image be $I_{r s}$ which is the restored approximation of the original image $I$.

\section{4. Signature Verification}

In order to preserve the low computational complexity, we reverse the RST attacks on demand. No affine transformation is occurred in non-geometric attacks like JPEG, noising etc. Moreover, in small rotations the transformation is insignificant in terms of MSB similarity rate as discussed in the motivation part. Let the attacked image be $I_{1}$. The signature is calculated for $I_{1}$ using the same procedure discussed in section 3.1 and 3.2 and compared with the previously calculated signature $S_{I L}$. If they do not match, the possible RST attack is reversed for $I_{1}$ in order to restore an approximate original using the procedure in section 3.3. The signature is again calculated for the obtained approximate original and compared with $S_{L L}$. If they still do not match, then $I_{1}$ is not verified.

\section{EXPERIMENTAL RESULTS}

We implemented the proposed MSB-based scheme and existing Torus-based [7] and VQ-based [9] schemes with MATLAB 7 and tested their robustness using all the watermarking benchmark images in [13] with different logos against many attacks including those in stirMark 4.0 [14]. Due to space limitations, however, only the results obtained using the Lena image signed by the Monash logo, as shown in Fig. 2(a)-(b), are presented.

Table I presents the comparative results in terms of the peak signal to noise ratio (PSNR) and the logo retrieval rate (LRR) of the retrieved logos for the proposed and existing schemes [7,9]. Results for the MSB-based scheme in Table I were obtained without reversing the RST attack and where necessary the attacked images were resized to the original size before finding the IC. The Torus-based scheme performed the worst due to torusmapping, as shown in Fig. 2(c). In most of the cases the PSNR and in all the cases the LRR of the retrieved logos by the proposed scheme were much higher than those by the VQ-based scheme. In 
Table II: Approximate restored original image and logo retrieval results for the proposed MSB-based scheme against various geometric attacks using Lena image and Monash logo

\begin{tabular}{l|c|c|c|c|c|c}
\hline \hline \multirow{2}{*}{ Attacks } & \multicolumn{2}{|c|}{$\begin{array}{c}\text { Image PSNR } \\
(\mathrm{dB})\end{array}$} & \multicolumn{2}{c|}{ Estimated } & \multicolumn{2}{c}{ MSB logo } \\
\cline { 2 - 7 } & $\begin{array}{c}\text { Before } \\
\text { restore }\end{array}$ & $\begin{array}{c}\text { After } \\
\text { restore }\end{array}$ & $\begin{array}{c}\text { Angle } \\
\left({ }^{\circ}\right)\end{array}$ & $\begin{array}{c}\text { Scale- } \\
\text { factor }\end{array}$ & $\begin{array}{c}\text { PSNR } \\
(\mathrm{dB})\end{array}$ & $\begin{array}{c}\text { LRR } \\
(\%)\end{array}$ \\
\hline Flipping $(x$-axis) & 13.57 & $\infty$ & $-^{4}$ & $-^{4}$ & $\infty$ & 100 \\
\hline Flipping $(y$-axis) & 12.49 & $\infty$ & $-^{4}$ & $-^{4}$ & $\infty$ & 100 \\
\hline Rotation $\left(45^{\circ}\right)$ & 8.51 & 23.85 & 44.22 & $-^{4}$ & 24.26 & 97 \\
\hline Rot.-crop $\left(60^{\circ}\right)$ & 10.43 & 13.35 & 60.05 & $-^{4}$ & 29.53 & 99 \\
\hline Rot.-scale $\left(15^{\circ}, 0.81\right)$ & 8.64 & 19.90 & 14.47 & 0.787 & 24.39 & 95 \\
\hline RSF $^{1}\left(15^{\circ}, 0.81, y\right)$ & 8.46 & 19.87 & 14.65 & 0.787 & 23.68 & 96 \\
\hline RSF $^{1}\left(75^{\circ}, 0.81, y\right)$ & 8.47 & 23.11 & 75.70 & 0.813 & 25.82 & 97 \\
\hline Scale $(0.50)$ & $--^{3}$ & $-{ }^{3}$ & 0.00 & 0.502 & 22.88 & 95 \\
\hline Scale $(2.00)$ & $--^{3}$ & $-{ }^{3}$ & 0.00 & 2.043 & 23.17 & 97 \\
\hline Translation ${ }^{2}$ & 10.32 & 13.54 & $-{ }^{4}$ & $-{ }^{4}$ & $\infty$ & 100 \\
\hline \hline
\end{tabular}

${ }^{1}$ Rot.-scale-flip: rotate, scale down and flip around $y$-axis;

${ }^{2}$ Translate center $(0,0)$ to $(-40,40) ;{ }^{3}$ Parameters not measured due to unmatched image sizes; ${ }^{4}$ Not necessary to measure.

the remaining few cases, as shown in italic font, the PSNR of the retrieved logos by the proposed scheme were lower. However, it is no way indicative to the superiority of the VQ-based scheme for these kinds of attacks because the logo quality degrades severely during the VQ- encoding, as shown in Fig. 2(d). As a consequence the PSNR and LRR remained almost unchanged irrespective of logos for both the existing schemes, which in turn made the signature verification ambiguous. Fig. 3 plots PSNR and LRR of the retrieved logos from Lena image, which was compressed with JPEG for different quality factors. While the proposed MSB-based scheme always offered much higher LRR than both the existing schemes and higher PSNR than the Torus-based scheme; it outperformed the VQ-based scheme in term of the PSNR when JPEG quality factor was greater than 10 . However, in the case of the MSB-based scheme, the PSNR, which is always greater than $15 \mathrm{~dB}$, along with the high LRR distinguished the logo retrieval instances unambiguously; while for the existing schemes the logo retrieval instances are ambiguous due to low LRR. This is consistent with the observations made in the motivation. Table II shows logo retrieval results for the proposed MSB-based scheme against various geometric attacks. The attacked images were restored to the approximate original before calculating the signature using the procedure described in section 3.3.

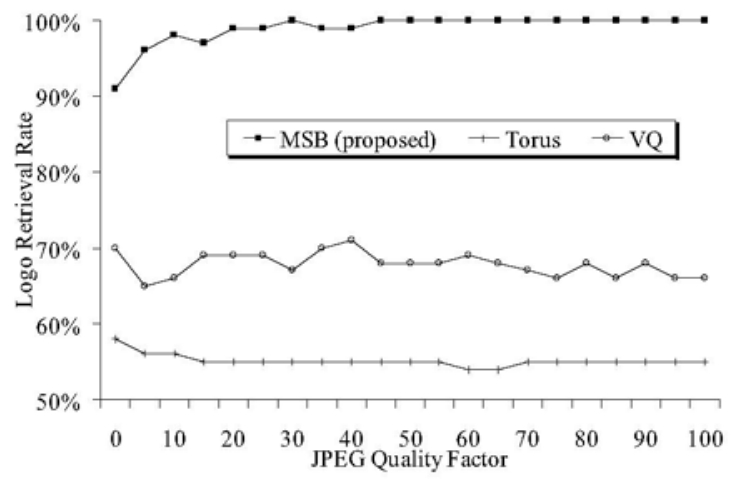

(a)

\section{CONCLUSIONS}

The proposed MSB-based copyright protection scheme offers the following advantages over the existing schemes: (i) any type and size of images and logos can be used; (ii) low computational complexity, due to use of exclusive-OR and low order geometric moments only, enables real time applications (for an image of size $N \times N$ and a logo of size $M \times M$, the time complexity by the Torusbased scheme is $O\left(n N M^{2}\right)$ where $n$ denotes the number of torusfunctions used for mapping; by the VQ-based scheme is $O\left(N^{2} M^{2}\right)$; and by the proposed MSB-based scheme is $O\left(N^{2}\right)$, i.e., any type and size of logos can be used with the same overhead); (iii) robust to different kinds of attacks; and (iv) RST attacked images are restored to the approximate original image before calculating the signature in verification phase. Although, for images, full of detailed textured and regular pattern and brightness change such as chessboard, the IC may not be converged to a unique point, real world images, in general, have not such compositions [10].

\section{REFERENCES}

[1] Bas, P., J. M. Chassery, and B. Macq, "Geometrically invariant watermarking using feature points," IEEE Trans. on Image Processing, 11(9), 1014-1028, 2002.

[2] Ruanaidh, O. and T. Pun, "Rotation, scale and translation invariant spread spectrum digital image watermarking," Signal Processing, 66(3), 303-317, 1998.

[3] Kim, H. S. and H. K. Lee, "Invariant image watermark using zernike moments," IEEE Trans. on Cir. \& Sys. for Vid. Tec., 13(8), 766-775, 2003.

[4] Awrangjeb, M. and M. S. Kankanhalli, "Reversible watermarking using a perceptual model," Electronic Imaging, 14(1), 013014-1-8, 2005.

[5] Chen, T. H., G. Horng, and W. B. Lee, "A publicly verifiable copyrightproving scheme resistant to malicious attacks," IEEE Trans. on Industrial Electronics, 52(1), 327-334, 2005

[6] Chang, C. C., K. F. Hwang, and M. S. Hwang, "Robust authentication scheme for protecting copyrights of images and graphics," in Proc. of Vision. Image and Signal Processing, 43-50, 2002

[7] Chang, C. C., J. Y. Hsiao, and C. L. Chiang, "An image copyright protection scheme based on torus automorphism," in Proc. of International Symposium on Cyber Worlds, 217-224, 2002

[8] Chang, C. C. and J. C. Chuang, "An image intellectual property protection scheme for gray-level images using visual secret sharing strategy," Pattern Recognition Letters, 23(8), 931-941, 2002.

[9] Lee, W. B. and T. H. Chen, "A public verifiable copy protection technique for still images," Systems and Software, 62(3), 195-204, 2002.

[10] Kim, B. S., J. G. Choi, C. H. Park, J. U. Won, D. M. Kwak, S. K. Oh, C. R. Koh, and K. H. Park, "Robust digital image watermarking method against geometrical attacks," Real Time Imaging, 9, 139-149, 2003.

[11] Alghoniemy, M and A. H. Tewfik, "Geometric invariance in image watermarking," IEEE Trans. on Image Processing, 13(2), 145-153, 2004.

[12] Free Fotot.com Inc., http://www.freefoto.com, Nov. 2005.

[13] http://abacus.ee.cityu.edu.hk/ benjiman/imagedb/, Sep. 2005

[14] Petitcolas, F. A. P., "Watermarking schemes evaluation," Signal Processing, $58-64,2000$.

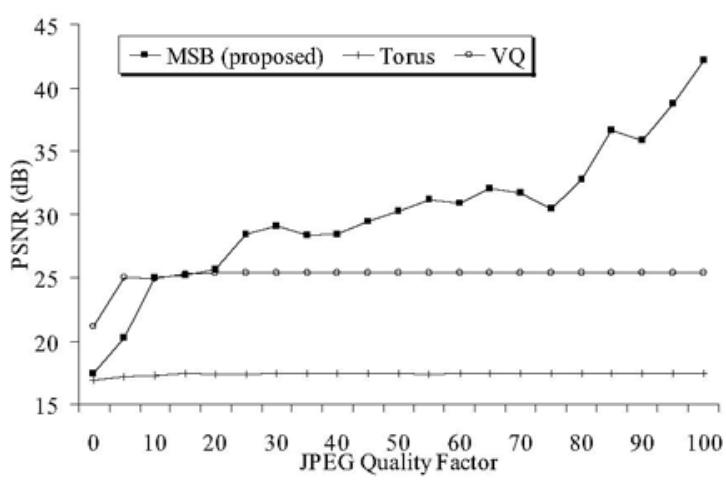

(b)

Fig. 3: (a) logo retrieval rate and (b) PSNR using Lena image and Monash logo by the proposed MSB-based scheme and existing Torus-based [7] and VQ-based [9] schemes against JPEG attacks for different quality factors 